\title{
Esgotamento entre profissionais da Atenção Primária à Saúde
}

\author{
Burnout Syndrome in Primary Health Care Professionals
}

Leonardo Fernandes Martins ${ }^{1}$

Tamires Jordão Laport ${ }^{1}$

Vinicius de Paula Menezes ${ }^{1}$

Priscila Bonfante Medeiros ${ }^{1}$

Telmo Mota Ronzani ${ }^{1}$

${ }^{1}$ Programa de PósGraduação em Psicologia, Universidade Federal de Juiz de Fora. R. José Lourenço Kelmer s/n Campus Universitário, São Pedro. 36036-900 Juiz de Fora MG Brasil.

telmo.ronzani@uff.edu.br
Abstract Burnout is characterized by emotional exhaustion, depersonalization and low occupational performance, which may occur among health professionals. This article evaluates burnout among workers in Primary Health Care (PHC) in three small towns in the Zona da Mata Mineira. The study analyzes associations by logistic regression between burnout, socioeconomic, and demographic aspects of work. A total of 149 professionals were selected, 107 of these responded to all questionnaires. To measure burnout, the Maslach Burnout Inventory (MBI) was used and to characterize the professional, a questionnaire assessing three different issues - namely individual and sociodemographic aspects and team area coverage - was used. 101 professionals were classified with positive indication for burnout. The variables present in the backward stepwise logistic regression model positively associated with indicative of burnout were: being younger than the population average ( $>29.5$ years) and use of drugs, including sedatives, tranquilizers and sleeping pills. The results contribute to the identification of factors associated with burnout and therefore highlight the need for more detailed investigation.

Key words Occupational burnout, Health personnel, Primary health care
Resumo O esgotamento profissional (EP) caracteriza-se por exaustão emocional, despersonalização, e baixa realização profissional, podendo ocorrer entre profissionais da saúde. Este artigo avalia o EP entre trabalhadores da Atenção Primária à Saúde (APS) em três municípios de pequeno porte da Zona da Mata Mineira. O estudo analisa a associação por regressão logística entre EP, aspectos socioeconômicos, demográficos e aspectos do trabalho. Um total de 149 profissionais foi selecionado, destes, 107 responderam a todos os questionários. Para mensurar o EP, foi utilizado o Maslach Burnout Inventory (MBI) e, para a caracterização dos profissionais, foi utilizado outro questionário segundo três diferentes questões: aspectos individuais, sociodemográficos e área de cobertura da equipe. Foram classificados 101 profissionais com indicação positiva para EP. As variáveis presentes no modelo de regressão logística backward stepwise, associadas positivamente com indicativo de esgotamento profissional foram: possuir idade inferior à mediana da população (> 29,5 anos) e fazer uso de medicamentos "calmantes, tranquilizantes e remédios para dormir". Os resultados contribuem para identificar fatores associados com o esgotamento profissional e apontam, neste sentido, para a necessidade de investigações mais detalhadas.

Palavras-chave Esgotamento profissional, Pessoal de saúde, Atenção Primária à Saúde 


\section{Introdução}

O termo esgotamento profissional começou a ser utilizado com maior regularidade no meio acadêmico e pelo senso comum a partir da década de 70, nos Estados Unidos. Nas décadas seguintes, este tema teve um importante destaque na literatura, principalmente entre estudos que avaliavam profissionais que trabalhavam em contato direto com o público ${ }^{1}$. O esgotamento profissional, em inglês Burnout, ganhou expressão e foi difundido através dos trabalhos seminais de Freunderberguer, em 1974, quando este usou o termo para descrever casos de gradual desgaste emocional, perda de motivação e reduzido comprometimento com o trabalho, entre profissionais de uma clínica de dependentes químicos de Nova York. Pouco tempo depois, Maslach começava, em 1976, a desenvolver uma conceituação formal do esgotamento profissional, utilizando a denominação Síndrome de Burnout para os quadros caracterizados pelos três aspectos apontados por Freunderberguer ${ }^{1,2}$.

Os primeiros estudos de Maslach obtiveram resultados empíricos acerca do esgotamento profissional adotando métodos de investigação observacionais e entrevistas em profundidade com diversos profissionais que prestavam serviços em contato direto com o público ${ }^{2}$. Posteriormente, associado a estes estudos, em 1981, Maslach e Jackson desenvolveram um instrumento estruturado com propriedades psicométricas para mensurar o nível de esgotamento profissional entre estes profissionais. Através de estudos de construção e validação os referidos autores chegaram à versão do questionário denominado de "Maslach Burnout Inventory" (MBI). Este instrumento baseiase em uma abordagem psicossocial que analisa o esgotamento profissional a partir de três dimensões independentes: (1) Exaustão Emocional ou perda de recursos emocionais para lidar com o trabalho; (2) Despersonalização ou desenvolvimento de atitudes negativas, de insensibilidade e de cinismo com aqueles que recebem o serviço prestado; (3) Falta de Realização Pessoal, ou tendência a avaliar o próprio trabalho de forma negativa, associado com sentimentos e avaliações de baixa autoestima profissional.

A predisposição para a apresentação de esgotamento profissional neste conjunto é apontada tanto pela literatura internacional acerca do estresse entre profissionais de saúde atuantes na atenção primária à saúde ${ }^{3-8}$, como nos poucos estudos encontrados na revisão da literatura nacional ${ }^{9-12}$. O esgotamento profissional teria consequências negativas em quatro âmbitos: (1) emocional; (2) cognitivo e (3) comportamental; e (4) social; os quais se relacionam com a saúde geral e qualidade de vida daqueles que padecem desta síndrome ${ }^{13}$. Alguns estudos apontam para índices de prevalências significativos da síndrome entre profissionais de saúde no Brasil ${ }^{14-17}$, inclusive entre profissionais da Estratégia de Saúde da Família (ESF) ${ }^{9,18}$.

A Atenção Primária à Saúde (APS) como uma estratégia complexa de promoção da saúde, prevenção de doenças e agravos, oferta de cuidados e reabilitação tem uma importância fundamental para a organização do sistema de saúde brasileiro. No modelo de APS brasileiro, operacionalizado através da ESF, os profissionais de saúde possuem um papel central, pois ao utilizarem tecnologias leves para resolução de problemas complexos necessitam estar em contato direto com pacientes e comunidades. Nesse sentido, os serviços de saúde na APS enquadram-se na categoria de profissões que lidam diretamente com demandas advindas de outras pessoas, exigindo que a própria execução do trabalho envolva o relacionamento interpessoal direto e contínuo com o beneficiado pelo serviço prestado, visando um cuidado integral que pode acabar por expor o profissional de saúde a importantes estressores psicossociais ${ }^{19}$.

A principal classe de estressores psicossociais apontada como mais característica do trabalho em saúde é a classe dos estressores ligados ao relacionamento interpessoal no trabalho ${ }^{20}$. Os profissionais de saúde acabam por ficar expostos a estes estressores, principalmente por terem que lidar diretamente com demandas complexas dos pacientes que atendem ${ }^{10,14,15,18,21,22}$. Não obstante, a exposição crônica aos estressores dessa natureza pode desencadear intensa exaustão emocional, redução da satisfação no trabalho e dificuldades para lidar com os pacientes de forma humanizada, configurando o quadro clínico denominado síndrome de Burnout ou, como adotado aqui, síndrome do esgotamento profissional ${ }^{23}$. Alguns estudos apontam que a cronicidade do esgotamento emocional pode ser o fator desencadeante para o surgimento deste quadro, que gera consequências negativas para a saúde individual e organização a que o profissional pertence ${ }^{15}$.

O esgotamento profissional aparece usualmente associado à diminuição da produção, da qualidade do trabalho executado, ao aumento do absenteísmo, aumento da rotatividade, bem como com o incremento de acidentes ocupacionais, o que, por fim, pode acarretar consideráveis prejuízos financeiros para as organizações e prejuízos 
para a própria saúde dos trabalhadores ${ }^{23}$. Considerando tais consequências, os fatores relacionados ao esgotamento profissional podem gerar danos diretos e indiretos aos pacientes atendidos.

Diante da ausência de estudos anteriores em municípios de pequeno porte e frente à grande variação entre os resultados apresentados na literatura nacional, o objetivo geral do presente estudo foi avaliar o esgotamento profissional entre trabalhadores da APS em três municípios de pequeno porte da Zona da Mata Mineira. Os objetivos específicos foram: (1) descrever a indicação de esgotamento profissional com base na identificação de alta exaustão emocional, alta despersonalização ou baixa realização pessoal; (2) analisar a existência de associação entre esgotamento profissional, aspectos socioeconômicos, demográficos e aspectos do trabalho.

\section{Método}

\section{Participantes}

Um total de 149 profissionais das APS, de três munícipios de pequeno porte de Minas Gerais, foram eleitos para participar deste estudo. Destes, 107 responderam a todos os questionários. Isto é, a taxa de resposta foi de 71,8\%, considerada muito boa para um levantamento ${ }^{24}$.

\section{Procedimentos e instrumentos}

Esta pesquisa foi desenvolvida com um caráter exploratório, possuindo objetivos descritivos e correlacionais. Devido ao caráter exploratório, propõe-se a investigação de associações entre o esgotamento profissional e as variáveis explicativas.

Para mensurar as variáveis propostas, foram utilizados instrumentos sigilosos e autoaplicáveis, sendo estes aplicados em duas seções. Para mensurar o esgotamento profissional foi utilizado o Maslach Burnout Inventory (MBI), um instrumento com propriedades psicométricas satisfatórias, traduzido e validado para a população brasileira por Lautert ${ }^{25}$ e Robayo-Tamayo ${ }^{16}$. Esse instrumento possui três dimensões, sejam elas, exaustão emocional, despersonalização e realização pessoal, contabilizando um total de 22 itens sendo respondidos numa escala de 1 a 5 . Os valores de confiabilidade calculados através do Alfa de Cronbach das escalas utilizadas no presente estudo foram os seguintes: Exaustão Emocional $=0,83$; Realização Pessoal =0,79; e Despersonalização $=0,49$.
Além deste questionário foi utilizado outro que não é considerado um instrumento psicométrico e tem por objetivo fazer uma caracterização dos profissionais e sua atuação segundo 3 diferentes grupos de questões: (1) Aspectos individuais (prática de atividades físicas; participação em culto religioso; uso de tranquilizantes ou calmantes para dormir; uso de tabaco e atividades de lazer); (2) Sociodemográficos (gênero; idade; profissão; prática religiosa; escolaridade máxima; renda familiar; profissão; tempo de serviço; treinamentos já recebidos; satisfação com o trabalho e atividades executadas fora do trabalho); (3) Área de cobertura da equipe (zona da área de atuação, percepção de riscos associados com o trabalho, característica de infraestrutura básica da área de cobertura e avaliação do principal problema da área de cobertura e perspectiva de aposentadoria).

\section{Análise de dados}

A população estudada foi considerada uma amostra aleatória simples e, dessa maneira, análises inferenciais foram realizadas e analisadas através do software Statistical Package for Social Science - SPSS ${ }^{\circledR}$. A análise foi dividida em duas fases: na primeira, o esgotamento profissional foi cruzado com todas as variáveis explicativas. $\mathrm{Na}$ segunda fase, as variáveis que apresentaram associações com esgotamento profissional foram utilizadas em conjunto para construírem um modelo explicativo para o esgotamento profissional.

Na primeira fase de preparação das análises, o indicativo positivo de esgotamento profissional foi categorizado com a resposta " $\operatorname{sim}=1$ " e o indicativo negativo foi categorizada como "não $=0$ ". O indicador adotado seguiu os critérios de Grunfeld et al. ${ }^{26}$, em que os participantes que apresentam escores acima do percentil 75 nas sub-escalas de exaustão emocional ou despersonalização são considerados com indicativos de esgotamento profissional, assim como aqueles que apresentam escores abaixo do percentil 25 para realização pessoal. Desconsiderando os casos faltantes, um total de 101 profissionais foi classificado através desse indicador, sendo que 41,6\% destes apresentaram indicação positiva para esgotamento profissional. O mesmo procedimento foi realizado para as demais variáveis explicativas.

Para a categorização das variáveis quantitativas, o ponto de corte utilizado foi a mediana; já para as variáveis qualitativas (não dicotômicas), utilizou-se uma categoria de referência (indicada na tabela). Para as questões semiestruturadas 
(percepção de risco e identificação de problemas no trabalho) as respostas foram categorizadas por dois pesquisadores, cada uma das categorias principais representou uma variável do tipo dicotômica, que indicava se o participante possuía em sua resposta uma das categorias.

A seguir, o esgotamento profissional foi testado quanto a correlações com seis tipos de variáveis, sendo estas: dados sociodemográficos, aspectos do trabalho, estilo de vida, infraestrutura básica, percepção de risco, e identificação dos principais problemas a serem resolvidos na área de atuação.

Quanto aos dados sociodemográficos, o esgotamento profissional foi correlacionado com as seguintes variáveis: 1) sexo, 2) idade, 3) possuir cônjuge, 4) escolaridade máxima, 5) estudar atualmente, 6) renda e 7) possuir filhos. As variáveis de estilo de vida correlacionadas com esgotamento profissional foram as seguintes: 1) lazer, 2) tabaco, 3) uso de medicamentos (ansiolíticos, hipnóticos, antidepressivos), 4) culto religioso, e 5) atividade física. As sete variáveis relativas à infraestrutura básica correlacionadas com o esgotamento profissional foram: 1) infraestrutura completa, 2) saneamento de esgoto, 3) pavimentação ou calçamento, 4) saneamento de água, 5) coleta de lixo, 6) acesso por transporte público, e 7) acesso a escola pública.

Um total de oito variáveis de percepção de risco foi associado com o esgotamento profissional, são elas: 1) trabalho arriscado, 2) contaminação de doenças, 3) animais, 4) lugares desertos, 5) área de tráfico de drogas/ marginalidade/ violência, 6) desgaste físico/emocional, 7) violência física/emocional, e 8) exposição solar.

A última variável correlacionada com o esgotamento profissional foi a identificação dos principais problemas a serem resolvidos na área de atuação. Para isso, nove variáveis foram analisadas, sendo elas: 1) identificação de pelo menos um problema a ser resolvido, 2) saneamento básico, 3) estrutura e organização do serviço, 4) transporte, 5) calçamento ou pavimentação, 6) problemas associados ao uso de álcool e outras drogas, 7) famílias carentes e pobres, 8) segurança pública (violência), e 9) desemprego.

O esgotamento profissional foi cruzado através de tabelas do tipo $2 \times 2$ com todas as variáveis explicativas para o cálculo do Odds Ratio (OR). O intervalo de confiança de $95 \%$ foi calculado para todos os valores estimados de OR. Para avaliar se estas relações entre as proporções observadas possuíam relacionamento estatisticamente significativo, foram calculados os valores de QuiQuadrado de Pearson para cada tabela e o valor de 'p' de cada associação, utilizando o teste exato de Fisher para os casos em que foram violadas as propriedades do Qui-Quadrado.

Para a construção do modelo explicativo de esgotamento profissional, as variáveis explicativas que apresentaram associações estatisticamente significativas com este e que possuíam valores de $\mathrm{p}<0,10$ foram selecionadas como covariáveis. Considerando a natureza dicotômica dos desfechos, optou-se por utilizar modelos de regressão logística para avaliar a capacidade preditiva das variáveis explicativas como covariáveis para esgotamento profissional. Utilizou-se esta técnica estatística também para identificar valores de significância e Odds Ratio ajustados de cada variável ao se controlar os efeitos das demais variáveis presentes no modelo.

Foi utilizado o modelo logístico de entrada de variáveis denominado Backward Stepwise, já para avaliação do ajuste do modelo aos dados utilizou-se o teste de Hosmer e Lemeshow. O teste de ajuste do modelo foi complementado pelo valor de $\mathrm{Rn}^{2}$ de Nagelkerke. Foi realizado também o teste de Omnibus para os coeficientes do modelo, adotando o valor de $\mathrm{p}<0,05$ para rejeição da hipótese nula. Adicionalmente foi avaliada a multicoliniariedade a fim de evitar sobre ajuste nos modelos.

Vale notar que, anteriormente a todo este processo de análises estatísticas, todo o banco de dados passou por um processo de dupla digitação e, após a comparação das duas versões, foi encontrada uma taxa de erro de $3 \%$. Todas as questões que possuíam erros de digitação foram corrigidas com base no questionário original.

\section{Aspectos éticos}

O projeto foi submetido e aprovado pelo Comitê de Ética em Pesquisa da Universidade Federal de Juiz de Fora. Todos os procedimentos adotados foram anteriormente negociados com as secretarias de saúde e coordenadores de atenção primária de cada município.

\section{Resultados}

Os resultados são apresentados através da descrição do esgotamento profissional e suas três dimensões, a saber: exaustão emocional, despersonalização, e realização pessoal. Além das associações entre estas e os aspectos investigados. Por fim, apresentam-se os modelos de regressão logística que propõem indicar os aspectos inves- 
tigados como variáveis explicativas do modelo do esgotamento profissional.

\section{Esgotamento profissional}

Em relação aos escores das três dimensões, foram encontrados para exaustão emocional na população $(\mathrm{n}=101)$ uma média de 20,30 (DP = $6,50)$, sendo o valor máximo 40 pontos e o mínimo 9 pontos. O critério de classificação utilizado adotou o percentil $25(16,00)$ e $75(24,00)$ como pontos de corte da distribuição. Foram considerados com níveis baixos de exaustão emocional $21,1 \%$ dos profissionais, com níveis médios $52,3 \%$ e considerados com níveis altos 20,6\%. A média dos escores para despersonalização encontrada na população estudada $(n=106)$ foi de $7,84(\mathrm{DP}=2,77)$, sendo o valor máximo 16 pontos e o mínimo 5 pontos. Foram considerados com níveis baixos de despersonalização 28,0\% dos participantes, apresentando níveis médios $50,0 \%$ e níveis altos $21,1 \%$. Já a média dos escores para realização pessoal $(\mathrm{n}=101)$ foi de 34,83 (DP $=5,09$, ) sendo o valor máximo 40 e o mínimo 18 . Foram considerados com níveis baixos de realização pessoal $21,5 \%$ dos profissionais, com níveis médios 55,4\% e níveis altos $20,6 \%$.

\section{Associações entre os fatores investigados e esgotamento profissional}

\section{Variáveis sociodemográficas}

A variável sexo apresentou associação significativa com o indicativo de esgotamento profissional ( $\mathrm{p}<0,01)$. Os homens apresentaram aproximadamente 2 vezes mais chances de possuírem uma classificação positiva quando comparados às mulheres. A idade também esteve associada com o esgotamento $(\mathrm{p}=0,05)$. Os profissionais que possuíam 30 anos ou mais revelaram 2,2 vezes menos chances de apresentarem esgotamento quando comparados com os que possuíam 29 anos ou menos. As demais variáveis sociodemográficas não apresentaram relacionamento estatisticamente significativo, indicando que escolaridade máxima, estudar atualmente, possuir renda acima de R\$ 1.300,00 e possuir filhos não estão associados ao esgotamento nesta população.

\section{Aspectos do trabalho}

Os profissionais que declararam que o trabalho na estratégia de saúde de família foi a sua primeira experiência profissional apresentaram cerca de 4 vezes mais chances de serem classificados com esgotamento profissional $(\mathrm{p}=0,01)$.
Por sua vez, os que declararam estarem satisfeitos ou muito satisfeitos no trabalho apresentaram $64 \%$ menos chances de estarem esgotados ( $\mathrm{p}=$ $0,03)$. As demais variáveis relacionadas com os aspectos do trabalho, elencados como ausência no trabalho por motivo de saúde, profissão (nível superior), atuar na área rural, ser treinado para atuar na ESF e tempo de trabalho na ESF, não apresentaram associação com o esgotamento profissional.

\section{Estilo de vida}

Nos cruzamentos bivariados o uso de medicamentos apresentou relacionamento significativo com a presença de esgotamento profissional $(\mathrm{p}<0,01)$. O uso de medicamentos ansiolíticos, hipnóticos e antidepressivos esteve associado a uma chance de aproximadamente 9:1 para a classificação positiva de esgotamento profissional, quando comparados aos que não utilizam esses tipos de medicamento ( $\mathrm{p}<0,01)$. Essa estimativa variou entre aproximadamente 2 vezes até 42 vezes mais chances de apresentar indicativo de esgotamento profissional. Os profissionais que relataram frequentar ao menos uma vez por semana algum tipo de culto religioso possuíam 3,8 vezes chances de serem classificadas como possíveis casos de esgotamento profissional, quando comparados ao grupo que não frequentava nenhum tipo de culto. As variáveis relacionadas ao estilo de vida tais como: lazer, tabaco e atividade física não apresentaram relacionamento estatisticamente significativo com o esgotamento profissional entre os profissionais de APS.

\section{Características ambientais}

\section{de vulnerabilidade}

Com exceção do acesso à escola pública e coleta de lixo, todos os demais fatores apresentaram-se associados significativamente com o esgotamento profissional. Os profissionais que atuavam em áreas que possuíam saneamento de esgoto em todos os seus setores apresentaram 2,3 vezes menos chances de estarem esgotados ( $\mathrm{p}=$ $0,01)$. O trabalho em locais com presença de calçamento ou pavimentação e saneamento de água foi também um fator de proteção, indicando que os profissionais dessas áreas têm 2,4 vezes menos chances de estarem esgotados (pavimentação ou calçamento, $\mathrm{p}=0,04$; saneamento de água, $\mathrm{p}=$ 0,05). Por fim, os trabalhadores dos locais onde se podia ter acesso aos meios de transporte público apresentaram 3,2 vezes menos chances de serem classificados como esgotados. O indicador de infraestrutura básica geral também esteve 
associado com o esgotamento profissional ( $\mathrm{p}=$ $0,05)$.

\section{Percepção de risco}

Nenhuma das variáveis relacionadas com a percepção de riscos no trabalho esteve associada com a presença de esgotamento profissional de forma significativa.

\section{Identificação dos principais problemas a serem resolvidos na área de atuação}

A identificação de algum problema a ser resolvido na área de atuação ou área de cobertura esteve associada com o esgotamento profissional $(\mathrm{p}=0,03)$. Os profissionais que identificaram que existe pelo menos um problema a ser resolvido apresentaram cerca de 3 vezes mais chances de apresentarem esgotamento profissional. A identificação do saneamento básico como principal problema a ser resolvido no setor de atuação esteve associada significativamente com o esgotamento profissional $(\mathrm{p}=0,02)$. Os profissionais que apontaram este como um dos principais problemas tiveram cerca de 3 vezes mais chances de apresentarem esgotamento profissional quando comparados aos demais. As demais variáveis relacionadas à identificação dos principais problemas das áreas de atuação não apresentaram associação com o esgotamento profissional, a saber: estrutura e organização do serviço, calçamento ou pavimentação, problemas associados ao uso de álcool e outras drogas, famílias carentes e pobres, segurança pública (violência), e desemprego.

As associações significativas entre as variáveis investigadas e o esgotamento profissional estão descritas na Tabela 1.

\section{Modelo explicativo de regressão logística para esgotamento profissional}

As variáveis que apresentaram níveis de associação significativa com o esgotamento profissional com $\mathrm{p}<0,01$ foram separadas em cada um dos seus grupos, formando blocos de variáveis a serem inseridas no modelo de regressão logística do tipo explicativo, tendo como desfecho o indicador de presença ou ausência de esgotamento profissional.

Inicialmente foi avaliada a existência de associação entre as variáveis explicativas a fim de evitar multicoliniariedade no modelo. Os principais problemas de multicoliniariedade foram encontrados entre as variáveis relacionadas à infraestrutura, sendo a solução para este problema utilizar o indicador de infraestrutura completa.
Ao adotar este último indicador, observou-se ainda que entre os principais problemas identificados com $\mathrm{p}<0,01$ (transporte, saneamento básico e identificação de pelo menos um problema) também estavam correlacionados o indicador de infraestrutura completa. Considerando que o indicador de existência de infraestrutura completa é um indicador mais objetivo, tendo sido obtido através de questões estruturadas, optou-se por utilizá-lo no modelo, retirando os problemas de transporte e saneamento básico.

Em relação à hipótese nula que mantém apenas a constante no modelo explicativo inicial para predizer a categoria com maior proporção, o modelo com todas as variáveis apresentou diferença estatisticamente significativa $\left(\chi^{2}=27\right.$, 225; $\mathrm{df}=10 ; \mathrm{p}<0,001)$, o que indicou que esse modelo é melhor preditor que apenas a constante da equação. A capacidade preditiva do modelo para identificar casos negativos de esgotamento profissional foi de $86,3 \%$ e sua capacidade preditiva para os casos positivos foi de $61,1 \%$, o que resulta em uma porcentagem média de acertos de $75,9 \%$, representando um aumento de $17,3 \%$ na porcentagem geral de acertos em relação à constante. $\mathrm{O}$ ajuste do modelo em relação aos dados mostrou-se adequado $\left(\mathrm{p}=0,63 ; \chi^{2}=6,168\right.$; DF $=8$, teste de Hosmer e Lemeshow), com a capacidade estimada de variância explicada de cerca de $36 \%\left(\mathrm{R}^{2}{ }_{\text {Nagelkerke }}=0,36\right)$.

A única variável presente no modelo, que apresentou valor de $\mathrm{p}<0,05$, foi o uso de remédios tranquilizantes, calmantes ou "para dormir", indicando que controlando o efeito das demais variáveis os profissionais que faziam uso destes medicamentos possuíam cerca de 9 vezes mais chances de serem classificados com esgotamento profissional (Tabela 2).

O modelo final, que buscou alcançar um maior ajuste dos dados em função das razões de verossimilhança das relações entre as variáveis, também apresentou diferença estatística em relação à hipótese nula $\left(\chi^{2}=20,371 ; \mathrm{df}=4 ; \mathrm{p}<\right.$ $0,01)$. Este modelo mostrou-se mais parcimonioso ao utilizar apenas 4 variáveis para alcançar uma capacidade preditiva geral de 71,3\% de classificação correta dos casos, diferença de 4,65\% com relação ao modelo com todas as variáveis. O modelo apresentou melhor ajuste aos dados do que o modelo inicial $\left(\chi^{2}=0,424 ; \mathrm{df}=4 ; \mathrm{p}\right.$ $=0,98$ no teste de Hosmer e Lemeshow), contudo, com uma menor explicação da variância de cerca de aproximadamente $28 \%\left(\mathrm{R}^{2}{ }_{\text {Nagelkerke }}=\right.$ $0,28)$. As variáveis explicativas que apresentaram significância estatística no modelo foram aqueles 
Tabela 1. Variáveis correlacionadas com o esgotamento profissional.

\begin{tabular}{|c|c|c|c|c|}
\hline \multirow[b]{2}{*}{ Variáveis } & \multicolumn{4}{|c|}{ Classificação positiva para esgotamento } \\
\hline & $\mathbf{n}$ & f & OR (IC95) & $\mathbf{p}^{*}$ \\
\hline Sexo (Masculino) & & & & 0,01 \\
\hline Não & 83 & 82,2 & 1 & \\
\hline Sim & 18 & 17,8 & $1,99(0,71-5,58)$ & \\
\hline Idade ( $>$ mediana 29,5 anos) & & & & 0,05 \\
\hline Não & 49 & 49,5 & 1 & \\
\hline Sim & 50 & 50,5 & $0,45(0,20-1,02)$ & \\
\hline Possui cônjuge & & & & 0,09 \\
\hline Não & 48 & 48,5 & 1 & \\
\hline Sim & 51 & 51,5 & $0,50(0,22-1,13)$ & \\
\hline Uso de medicamentos (ansiolíticos, hipnóticos, antidepressivos) & & & & 0,01 \\
\hline Não & 88 & 88,2 & 1 & \\
\hline Sim & 12 & 12,0 & $8,75(1,80-42,44)$ & \\
\hline Satisfeito no trabalho & & & & 0,03 \\
\hline Não & 23 & 22,8 & 1 & \\
\hline Sim & 78 & 77,2 & $0,36(0,14-0,94)$ & \\
\hline $1^{\circ}$ emprego na ESF & & & & 0,01 \\
\hline Não & 84 & 83,2 & 1 & \\
\hline Sim & 17 & 16,8 & $4,32(1,39-13,44)$ & \\
\hline Infraestrutura completa & & & & 0,05 \\
\hline Não & 58 & 57,4 & 1 & \\
\hline Sim & 43 & 42,6 & $0,43(0,19-0,99)$ & \\
\hline Saneamento de esgoto & & & & 0,01 \\
\hline Não & 34 & 33,7 & 1 & \\
\hline Sim & 67 & 66,3 & $0,34(0,15-0,80)$ & \\
\hline Pavimentação ou calçamento & & & & 0,04 \\
\hline Não & 32 & 31,7 & 1 & \\
\hline Sim & 69 & 68,3 & $0,42(0,18-0,98)$ & \\
\hline Saneamento de água & & & & 0,05 \\
\hline Não & 30 & 29,7 & 1 & \\
\hline Sim & 71 & 70,3 & $0,42(0,17-0,99)$ & \\
\hline Acesso por transporte público & & & & 0,02 \\
\hline Não & 22 & 21,8 & 1 & \\
\hline Sim & 79 & 78,2 & $0,31(0,12-0,84)$ & \\
\hline Identificação problema a ser resolvido & & & & 0,03 \\
\hline Não & 26 & 25,7 & 1 & \\
\hline Sim & 75 & 74,3 & $3,08(1,11-8,52)$ & \\
\hline Saneamento básico & & & & 0,02 \\
\hline Não & 81 & 80,2 & 1 & \\
\hline Sim & 20 & 19,8 & $3,33(1,2-9,28)$ & \\
\hline
\end{tabular}

*Valor de p calculado utilizando o teste do Qui-Quadrado de Pearson, com correção exata de Fisher para os cruzamentos com células que apresentaram frequências esperadas menores que cinco.

Fonte: do autor.

que demonstraram possuir idade inferior a mediana da população ( $>29,5$ anos), sendo que, ao controlar as demais variáveis no modelo, aqueles que possuíam as maiores idades apresentaram 2,7 vezes menos chances de serem classificados com esgotamento profissional. Seguindo uma direção contrária, aqueles que faziam uso de medicamentos apresentaram cerca de 9 vezes mais chances de serem classificados com esgotamento profissional. Participar de cultos religiosos ao menos uma vez por semana e este ser o seu primeiro emprego permaneceram no modelo como variáveis de ajuste (Tabela 3 ).

\section{Discussão}

A classificação de esgotamento profissional apontou 41,6\% dos profissionais com indicação positiva. Os dados da literatura referentes a es- 
Tabela 2. Valores ajustados de Odds Ratio para as variáveis associadas com o indicador de esgotamento profissional através do modelo de regressão logística com entrada de todas as variáveis em conjunto $(\mathrm{n}=87)$.

\begin{tabular}{lccccc}
\hline Método de entrada de todas variáveis & B & Erro Padrão & OR $_{\text {ajustado }}$ & IC 95\% & $\mathbf{p}$ \\
\hline Sociodemográficas & & & & & \\
$\quad$ Sexo (Masculino) & 0,58 & 0,72 & 1,79 & $0,44-7,28$ & 0,42 \\
$\quad$ Idade (> mediana 29,5anos) & $-0,67$ & 0,64 & 0,51 & $0,15-1,80$ & 0,30 \\
$\quad$ Possui cônjuge & $-0,30$ & 0,58 & 0,74 & $0,24-2,29$ & 0,60 \\
Aspectos do trabalho & & & & & \\
$\quad$ Emprego na ESF & 1,10 & 0,69 & 2,99 & $0,78-11,53$ & 0,11 \\
$\quad$ Satisfeito no trabalho & $-0,71$ & 0,72 & 0,49 & $0,12-2,01$ & 0,32 \\
$\quad$ Aposentaria na ESF & $-0,25$ & 0,62 & 0,78 & $0,23-2,63$ & 0,69 \\
$\quad$ Estilo de Vida & $-0,71$ & 0,57 & 0,49 & $0,16-1,50$ & 0,21 \\
$\quad$ Culto religioso & 2,24 & 0,97 & 9,43 & $1,41-63,19$ & $0,02^{*}$ \\
$\quad$ Uso de remédios & & & & & 0,24 \\
Percepção de Riscos & $-0,87$ & 0,73 & 0,42 & $0,10-1,77$ & 0,24 \\
$\quad$ Animais & & & & & 0,12 \\
Infraestrutura & $-0,93$ & 0,59 & 0,40 & $0,12-1,27$ & 0,12 \\
$\quad$ Infraestrutura completa & 1,25 & 0,80 & 3,50 & & \\
$\quad$ Constante &
\end{tabular}

Modelo apresentou ajuste adequado aos dados $\left(\mathrm{p}=0,63 ; \mathrm{x}^{2}=6,168 ; \mathrm{df}=8\right.$, teste de Hosmer e Lemeshow); $\mathrm{R}^{2}{ }_{\text {Nagelkerke }}=0,36{ }^{*}$ valor de $\mathrm{p}<0,05$.

Fonte: do autor.

Tabela 3. Valores ajustados de Odds Ratio para as variáveis associadas com o indicador de esgotamento profissional através do modelo de regressão logística backward stepwise para maximização das razões de verossimilhança $(\mathrm{n}=87)$.

\begin{tabular}{|c|c|c|c|c|c|}
\hline Método Backward Stepwise (razão de verossimilhança) & B & Erro Padrão & $\mathrm{OR}_{\text {ajustado }}$ & IC $95 \%$ & $\mathbf{p}$ \\
\hline \multicolumn{6}{|l|}{ Sociodemográficas } \\
\hline Idade ( $>$ mediana 29,5 anos) & $-1,01$ & 0,52 & 0,37 & $0,13-1,01$ & 0,05 \\
\hline \multicolumn{6}{|l|}{ Aspectos do trabalho } \\
\hline $1^{\circ}$ emprego na ESF & 1,10 & 0,65 & 3,00 & $0,83-10,82$ & 0,09 \\
\hline \multicolumn{6}{|l|}{ Estilo de vida } \\
\hline Culto religioso & $-0,94$ & 0,52 & 0,39 & $0,14-1,08$ & 0,07 \\
\hline Uso de remédios & 2,21 & 0,90 & 9,14 & $1,57-53,15$ & 0,01 \\
\hline Constante & 0,27 & 0,53 & 1,31 & & 0,61 \\
\hline
\end{tabular}

Modelo apresentou ajuste adequado aos dados $\left(\mathrm{p}=0,98 ; \mathrm{x}^{2}=0,424 ; \mathrm{df}=4\right.$, teste de Hosmer e Lemeshow); $\mathrm{R}^{2}{ }_{\text {Nagelkerke }}=0,28$ * valor de $\mathrm{p}<0,05$.

Fonte: do autor.

sas classificações entre profissionais de saúde variam muito entre diferentes classes profissionais e dentre diferentes critérios de correção adotados para avaliação do esgotamento. O critério adotado neste estudo é considerado o mais abrangente por identificar indícios da síndrome a partir das classificações altas em qualquer uma das três subescalas do MBI, sendo considerado um critério de rastreio para a síndrome de esgotamento profissional ${ }^{26}$.
Os estudos entre profissionais de saúde do Brasil, que utilizaram o critério adotado, encontraram entre médicos cancerologistas de 52,3\% a $68,6 \%$ de indicação positiva para esgotamento profissional ${ }^{27,28}$, entre médicos intensivistas $63,3 \%{ }^{29}$ e $35,7 \%$ entre enfermeiros de hospitais gerai $^{30}$. O único estudo realizado na estratégia de saúde da família entre agentes comunitários de saúde, que utilizou um indicador de esgotamento profissional, adotou o critério mais restrito de 
classificação e encontrou o indicativo da síndrome de esgotamento profissional de $24,1 \%$ entre os agentes comunitários ${ }^{9}$.

A diferença entre estas proporções poderia estar relacionada à própria caracterização dos municípios de pequeno porte, os quais poderiam oferecer mais recursos de enfrentamento de situações estressoras, mas também podem ser fruto da influência das outras categorias profissionais que compõem a população estudada aqui. Ao comparar os indicativos de esgotamento profissional, percebe-se que o encontrado neste estudo é menor em comparação ao dos médicos atuantes em serviços especializados ${ }^{27-29}$ e maior do que entre os enfermeiros de hospitais gerais ${ }^{30}$.

As variáveis explicativas que apresentaram relacionamento estatisticamente significativo com o esgotamento profissional, presentes no modelo de melhor ajuste apresentado, foram idade e o uso de "medicamentos calmantes, tranquilizantes ou remédios para dormir”. Este último, esteve associado com um risco de cerca de 9 vezes maior de classificação positiva para esgotamento profissional, o que pode indicar uma associação indireta com transtornos psiquiátricos não psicóticos, os quais são frequentemente associados com a síndrome de esgotamento.

Virtanen et al. ${ }^{31}$, utilizando dados relativos à prescrição de antidepressivos para a população geral, encontrou forte associação do seu uso com indicadores de estresse no trabalho. Em estudos futuros seria importante avaliar diferentes classes de substâncias psicotrópicas utilizadas com ou sem prescrição médica a fim de melhor compreender tal associação, inclusive adotando informações mais seguras como análise de prontuários médicos.

Os profissionais com idades entre 30 ou mais anos apresentaram 2,7 vezes menos chances de serem classificados com síndrome de esgotamento profissional. Uma das explicações para o efeito protetor da idade em estudos acerca da saúde do trabalhador é denominado o "efeito do trabalhador sadio", que considera que profissionais mais velhos têm mais chances de se encontrarem ativos e saudáveis, pois os profissionais que adoecem no trabalho acabam abandonando seus postos, sendo afastados do serviço ou mesmo mudando de área de atuação ${ }^{32}$. A literatura também aponta a mesma relação em outros estudos acerca do esgotamento profissional entre profissionais de saúde 9,29,33. $^{2}$

Apesar de não significativa no modelo final, a variável que avaliava se o primeiro emprego do profissional foi na ESF permaneceu como variá- vel de ajuste possuindo um valor de Beta negativo, o que parece indicar um menor risco para o esgotamento entre os profissionais que já trabalharam em outros empregos. Dessa forma, esse dado também pode ser associado com a hipótese do "trabalhador saudável”, já que os profissionais que têm como seu primeiro emprego o trabalho na ESF têm, em geral, a tendência de serem mais jovens e possuírem menos tempo de serviço, o que os predisporia a apresentarem mais problemas de saúde associados ao trabalho. Contudo, estudos apontam que a possibilidade de esgotamento profissional em situações de primeiro emprego pode estar associada à frustração advinda da realidade da prática que contrastam com conteúdos aprendidos durante a formação ${ }^{34}$.

A participação em cultos religiosos, ao menos uma vez por semana, apesar de não apresentar relacionamento estatisticamente significativo no modelo final, parece indicar que a frequência a cultos religiosos é um fator de proteção para o indicativo do esgotamento, o qual se apresenta consoante com o estudo entre médicos cancerologistas no Brasil ${ }^{27}$. Além disso, Yi et al. ${ }^{35}$ encontraram entre residentes de medicina atuantes na atenção primária, que a não utilização de estratégias de enfrentamento baseadas na religiosidade e que o sentimento de pobre bem-estar associado com a espiritualidade são preditores importantes para depressão. O referido autor aponta em suas conclusões a importância de se desenvolver treinamento que evidenciem a utilização de recursos de enfrentamento baseados na espiritualidade.

No presente estudo, os indicadores utilizados não permitem apontar para prejuízos diretos associados ao esgotamento profissional, mas evidenciam possíveis problemas. Como por exemplo, os resultados relativos aos maiores índices de esgotamento profissional associados com o uso de "medicamentos calmantes", chama muito a atenção, principalmente pelo indicativo de possíveis problemas psiquiátricos associados.

Como problema adicional, como em todo estudo correlacional, é possível que as associações observadas sofram o efeito de terceiras covariáveis, mesmo adotando as técnicas de análise de multicoliniariedade para evitar sobreajuste no modelo. O recurso de levantar inicialmente um grande número de variáveis explicativas a partir de diversos aspectos buscou minimizar esse tipo de viés.

Uma cautela a ser levada em conta para a interpretação dos resultados deve-se ao tamanho da amostra utilizada, os modelos de regressão logística, como outras técnicas estatísticas, são sen- 
síveis às variações no tamanho das amostras estudadas. Tamanhos amostrais pequenos podem gerar estimativas de significância subestimada e valores de intervalo de confiança para as análises do Odds Ratio extremamente altos.

Cabe ressaltar, que mesmo considerando o objetivo exploratório do estudo, é importante verificar que o critério de intencionalidade de seleção da população estudada, a própria participação voluntária e o tamanho pequeno do " $n$ " final adotado, diminuem as possibilidades de generalização dos resultados, apesar de servirem como bons indícios de levantamento de hipóteses a serem testadas com delineamentos mais robustos.

No entanto, a relevância deste estudo fica maior considerando que $90 \%$ dos mais de cinco mil municípios brasileiros possuem menos do que 50 mil habitantes e, destes, $48 \%$ possuem menos do que 10 mil habitantes e organizam seus serviços de modo semelhante ao encontrado nos municípios estudados ${ }^{36}$.

\section{Considerações finais}

O presente estudo, como todo estudo exploratório, aponta mais indícios e questões do que respostas para os fenômenos estudados. O desenvolvimento de estudos futuros abordando as lacunas apontadas pode contribuir muito para o avanço do tema. Considerando as consequências negativas do esgotamento profissional, buscar subsídios para entender e diminuir os problemas a este associados é uma forma de também avançar na melhoria do cuidado ofertado aos profissionais e usuários, assim como contribuir para a implementação efetiva das práticas de atenção primária no país e de uma maior humanização do trabalho em saúde.
Estudos futuros precisam verificar melhor as relações encontradas, principalmente através da utilização de instrumentos que possam melhor mensurar as variáveis explicativas adotadas no presente estudo. Diversos fatores não foram abarcados como variáveis explicativas ou mesmo moderadoras, tais como a interface família-trabalho, suporte social e as estratégias de enfrentamento utilizadas pelos participantes. Além destes últimos fatores, outros desfechos podem ser melhores estudados, principalmente aqueles que são considerados como influenciados pelo esgotamento profissional, como é o caso de indicadores de saúde e satisfação dos usuários atendidos.

\section{Colaboradores}

LF Martins e TM Ronzani trabalharam na concepção e no delineamento, análise e interpretação dos dados, na redação do artigo e sua revisão crítica, e na aprovação da versão a ser publicada. TJ Laport, PB Medeiros e VP Menezes participaram da análise e interpretação dos dados, redação do artigo e na sua revisão crítica, e aprovação da versão a ser publicada.

\section{Agradecimentos}

Fundação de Amparo à Pesquisa de Minas Gerais (FAPEMIG); Conselho Nacional de Desenvolvimento Científico e Tecnológico (CNPq); Coordenação de Aperfeiçoamento de Pessoal de Nível Superior (Capes); Bolsa de produtividades em Pesquisa CNPq. 


\section{Referências}

1. Maslach C, Schaufeli WB, Leiter MP. Job burnout. Annu Rev Psychol 2001; 52(1):397-422.

2. Schaufeli WB, Leiter MP, Maslach C. Burnout: 35 years of research and practice. Career Development International 2008; 14(3):204-220.

3. An PG, Rabatin JS, Manwell LB, Linzer M, Brown RL, Schwartz MD, MEMO investigators. Burden of difficult encounters in primary care: data from the minimizing error, maximizing outcomes study. Arch Intern Med 2009; 169(4):410-414.

4. Arigoni F, Bovier PA, Mermillod B, Waltz P, Sappino AP. Prevalence of burnout among Swiss cancer clinicians, paediatricians and general practitioners: Who are most at risk? Support Care Cancer 2009; 17(1):75-81.

5. Haq Z, Iqbal Z, Rahman A. Job stress among community health workers: A multi-method study from Pakistan. International Journal of Mental Health Systems 2008; 2:15.

6. Kushnir T, Cohen AH. Positive and negative work characteristics associated with burnout among primary care pediatricians. Pediatr Int 2008; 50(4):546-551.

7. Linzer M, Manwell LB, Williams ES, Bobula JA, Brown RL, Varkey AB. Working conditions in primary care: physician reactions and care quality. Ann Intern Med 2009; 151(1):28-36.

8. Virtanen P, Oksanen T, Kivimaki M, Virtanen M, Pentti J, Vahtera J. Work stress and health in primary health care physicians and hospital physicians. Occup Environ Med 2008; 65(5):364-366.

9. Silva ATC, Menezes PR. Esgotamento profissional e transtornos mentais comuns em agentes comunitários de saúde. Rev Saude Publica 2008; 42(5):921-929.

10. Feliciano KVO, Kovacs MH, Sarinho SW. Burnout entre médicos da Saúde da Família: os desafios da transformação do trabalho. Cien Saude Colet 2011; 16(8):3373-3382.

11. Suehiro ACB, Santos AAA, Hatamoto CT, Cardoso MM. Vulnerabilidade ao estresse e satisfação no trabalho em profissionais do Programa de Saúde da Família. Bol Psicol 2008; 58(129):205-218.

12. Trindade LL, Lautert L, Beck CLC. Coping mechanisms used by non-burned out and burned out workers in the family health strategy. Rev Lat Am Enfermagem 2009; 17(5):607-612.

13. Adán JCM, Jiménez BM, Herrer MG. Desgaste profesional y salud de los profesionales médicos: revisión y propuestas de prevención. Med Clin (Barc) 2004; 123(7):265-270.

14. Borges LO, Argolo JCT, Baker MCS. Os Valores Organizacionais e a Síndrome de Burnout: Dois Momentos em uma Maternidade Pública. Psicologia: Reflexão e Crítica 2006; 19(1):34-43.

15. Borges LO, Argolo JCT, Pereira ALS, Machado EAP, Silva WS. A Síndrome de Burnout e os Valores Organizacionais: Um Estudo Comparativo em Hospitais Universitários. Psicologia: Reflexão e Crítica 2002; 15(1):189-200.

16. Robayo-Tamayo M. Relação entre a Sindrome de Burnout e os valores organizacionais no pessoal de enfermagem de dois hospitais públicos [dissertação não publicada]. Brasília: Universidade de Brasília; 1997.
17. Lima RAS, Souza AI, Galindo RH, Feliciano KVO. Vulnerabilidade ao burnout entre médicos de hospital público do Recife. Cien Saude Colet 2013; 18(4):10511058.

18. Trindade LL. O estresse laboral da equipe de saúde da família: implicações para saúde do trabalhador [dissertação]. Porto Alegre: Universidade Federal do Rio Grande do Sul; 2007.

19. Carlotto MS, Câmara SG. Propriedades psicométricas do Maslach Burnout Inventory em uma amostra multifuncional. Estudos de Psicologia (Campinas) 2007; 24(3):325-332.

20. Rout UR, Rout JK. Stress Management for Primary Health Care Professionals. New York: Kluwer Academic Publishers; 2002.

21. Paschoalini B, Oliveira MM, Frigério MC, Dias ALRP, Santos FH. Efeitos cognitivos e emocionais do estresse ocupacional em profissionais de enfermagem. Acta Paulista de Enfermagem 2008; 21(3):487-492.

22. Santos AFO, Cardoso CL. Profissionais de saúde mental: manifestação de stress e burnout. Estudos de Psicologia (Campinas) 2010; 27(1):67-74.

23. Benevides-Pereira AMT. O Estado da Arte do Burnout no Brasil. Interação Psi 2003; 1(1):4-11.

24. Babbie E. Métodos de pesquisa de survey. Belo Horizonte: UFMG; 1999.

25. Lautert L. O desgaste profissional do enfermeiro [tese]. Salamanca: Universidade Pontífica de Salamanca; 1995.

26. Grunfeld E, Whelan TJ, Zitzelsberger L, Willan AR, Montesanto B, Evans WK. Cancer care workers in Ontario: prevalence of burnout, job stress and job satisfaction. CMAJ 2000; 163(2):166-169.

27. Glasberg J, Horiuti L, Novais MAB, Canavezzi AZ, Miranda VC, Chicoli FA, Gonçalves MS, Bensi CG, Giglio A. Prevalence of the burnout syndrome among Brazilian medical oncologists. Rev Assoc Med Bras 2007; 53(1):85-89.

28. Tucunduva LTCM, Garcia AP, Prudente FVB, Centofanti G, Souza CM, Monteiro TA, Vince FAH, Samano EST, Gonçalves MS, Del Giglio A. A síndrome da estafa profissional em médicos cancerologistas brasileiros. Rev Assoc Med Bras 2006; 52(2):108-112.

29. Barros DS, Tironi MOS, Nascimento Sobrinho CL, Neves FS, Bitencourt AGV, Almeida AM, Souza YGS, Teles MS, Feitosa AIR, Mota ICC, França J, Borges LG, Lordão MBJ, Trindade MV, Almeida MBT, Marques Filho ES, Reis EJFB. Médicos plantonistas de unidade de terapia intensiva: perfil sócio-demográfico, condições de trabalho e fatores associados à síndrome de burnout. Revista Brasileira de Terapia Intensiva 2008; 20(3):235240.

30. Moreira DS, Magnago RF, Sakae TM, Magajewski FRL. Prevalência da síndrome de burnout em trabalhadores de enfermagem de um hospital de grande porte da Região Sul do Brasil. Cad Saúde Pública 2009; 25(7):15591568.

31. Virtanen M, Honkonen T, Kivimaki M, Ahola K, Vahtera J, Aromaa A, Lönnqvist J. Work stress, mental health and antidepressant medication findings from the Health 2000 Study. J Affect Disord 2007; 98(3):189-197. 
32. Martinez MC. Estudo dos fatores associados à capacidade para trabalho em trabalhadores do setor elétrico [tese]. São Paulo: Universidade de São Paulo; 2006.

33. Kruse GR, Chapula BT, Ikeda S, Nkhoma M, Quiterio N, Pankratz D, Mataka K, Chi BH, Bond V, Reid SE. Burnout and use of HIV services among health care workers in Lusaka District, Zambia: A cross-sectional study. Human Resources for Health 2009; 7:55.

34. KVO, Kovacs MH, Sarinho SW. Sentimentos de profissionais dos serviços de pronto-socorro pediátrico: reflexões sobre o burnout. Revista Brasileira de Saúde Materno Infantil 2005; 5(3):319-328.

35. Yi MS, Luckhaupt SE, Mrus JM, Mueller CV, Peterman AH, Puchalski CM, Tsevat J. Religion, spirituality, and depressive symptoms in primary care house officers. Ambulatory Pediatrics 2006; 6(2):84-90.

36. Ministério da Saúde (MS), Organização Pan-Americana da Saúde. Sistema de Planejamento do SUS (PlanejaSUS): uma contrução coletiva. Brasília: MS; 2009.

Artigo apresentado em 04/04/2013

Aprovado em 07/10/2013

Versão final apresentada em 12/10/2013 